\title{
Un modelo para representar el estado anímico de los estudiantes en el aula*
}

\author{
Elena del $\mathrm{Val}^{1,2}$, Juan M. Alberola ${ }^{1,2}$ y Bexy Alfonso ${ }^{2}$ \\ ${ }^{1}$ Universitat Politècnica de València \\ ${ }^{2}$ Florida Universitària
}

\begin{abstract}
Students' affective state has influence in their learning process. Specifically, a positive state has positive consequences in aspects such as motivation and achievements. Even though the affective state is represented in the long-term, this can be modified through the emotions that emerge during the classes. In this paper, we present a model to represent the students' affective state in order to identify those factors that are related to positive affective states. Considering this model, we collected students' emotions during different lessons in different subjects. The results show that lessons that are more related to positive affective states are those oriented to working on projects and those that revise the previous content.
\end{abstract}

Keywords: affective state, students, emotions.

\section{Resumen}

El estado anímico de los estudiantes tiene una influencia directa en el proceso de enseñanza-aprendizaje. En concreto, un estado positivo permite mejorar aspectos como la motivación y los logros conseguidos. A pesar de que el estado anímico representa un estado a largo plazo, éste puede ser modificado a través de las emociones que tienen los estudiantes en función de las clases. En este artículo, presentamos un modelo para representar el estado anímico de los estudiantes con la finalidad de identificar qué factores influyen para conseguir estados positivos que faciliten su aprendizaje. Considerando este modelo, hemos recogido resultados midiendo las emociones que tienen los alumnos durante las distintas sesiones de varias asignaturas. Los resultados demuestran que las clases que generan más emociones positivas son aquellas en donde se trabaja por proyectos y en las que se repasan conceptos anteriores.

Keywords: estado anímico, estudiantes, emociones.

\footnotetext{
*Proyecto financiado por la Universitat Politècnica de València
} 


\section{Introducción}

El estado anímico de los estudiantes representa un rol muy importante en la educación debido a su influencia en los procesos cognitivos y comportamientos que tienen sobre las personas (Mehrabian 1996, Ryckman 2007, Chalfoun et al. 2006). En un entorno educativo, esto significa que un estado anímico positivo tiene consecuencias positivas sobre el proceso de enseñanza-aprendizaje (mayor motivación, mejores logros, mejor bienestar general, etc.). Por tanto, si podemos orientar nuestras estrategias educativas para conseguir unos estados anímicos más positivos en los estudiantes, mejorarán las posibilidades de mejorar aspectos como la satisfacción, el porcentaje de comprensión, la rapidez de adquisición de conocimiento o la motivación.

Modificar el estado anímico implica actuar sobre un conjunto de características que representan un estado a largo plazo. Sin embargo, las emociones son estudiadas como respuestas a estímulos (Ortony et al. 1988, Lazarus 1994, Scherer 2001). Por tanto, si podemos adaptar las estrategias educativas para conseguir producir emociones positivas, el estado anímico a largo plazo de los estudiantes tendería a mejorar, con el correspondiente resultado positivo sobre el proceso de enseñanza-aprendizaje.

Las emociones deben ser analizadas desde una perspectiva multidimensional, en la cuál se mida cómo varían las emociones entre los estudiantes, dependiendo de distintos factores. Por ejemplo, deberemos de analizar si la variación de las emociones puede explicarse por variables como las diferencias académicas entre las actividades, por las metodologías, por los tipos de clase, por los tipos de asignaturas, etc. Estos aspectos son fundamentales para diseñar correctamente intervenciones educativas (Pekrun et al. 2007).

En este artículo proponemos un modelo para representar el estado anímico de los estudiantes con la finalidad de observar el impacto que tienen diferentes estrategias educativas en el mismo. Las consideraciones prácticas sobre cómo podemos conseguir emociones positivas en los estudiantes podemos extraerlas a través del análisis de las observaciones de clases. En nuestro enfoque, presentamos un proceso que nos permite capturar las emociones que producen en los estudiantes los distintos eventos que ocurren en las clases. Para validar este modelo, presentamos datos sobre su aplicación práctica en varias asignaturas durante un curso académico.

El artículo se organiza de la siguiente manera. En la Sección 2 describimos el modelo propuesto para representar el estado anímico de los estudiantes. En la Sección 3 definimos el proceso para obtener información relativa a los estados de los estudiantes en las distintas clases. En la Sección 4 presentamos algunos datos sobre la evaluación del modelo en un entorno real. Finalmente, en la Sección 5 remarcamos las conclusiones principales del trabajo y orientaciones futuras. 


\section{Modelo del estado anímico de los estudiantes}

El modelo propio que proponemos para representar el estado anímico de los estudiantes (Figura 1) tiene en consideración un conjunto de circunstancias que influyen en el mismo y por tanto, en los factores de aprendizaje que queremos evaluar. Este conjunto de circunstancias se compone de tres dimensiones: individual, entorno, y social. Para cada dimensión, consideramos dos tipos de características: las que son estáticas y mantienen un valor fijo durante un período largo de tiempo y no suelen cambiar (un curso, un año académico, un semestre, etc.) y las que son dinámicas, que suelen cambiar cona frecuencia más alta (p.e. cada clase). En este sentido, aspectos como el horario de una asignatura se definirían como características estáticas puesto que suelen ser los mismos a lo largo del curso, mientras que aspectos como el tipo de clase se definirían como características dinámicas, puesto que puede haber una clase que utilice una metodología de lección magistral y otra puede trabajarse por equipos.

La dimensión individual representa el conjunto de características que tienen que ver con un estudiante individual y está compuesta de dos características estáticas: intereses y personalidad. Los intereses representan los objetivos personales del estudiante a largo plazo (p.e. aprender lo máximo posible, satisfacción personal, realizar el mínimo esfuerzo para aprobar, hacerlo bien en los exámenes para tener una buena nota, etc.). La relación entre personalidad y aprendizaje está ampliamente documentada en la literatura (Ibrahimoglu et al. 2013). En nuestro caso, consideramos los rasgos de personalidad definidos por el modelo Big Five para caracterizar la personalidad de los estudiantes (Costa y McCrae 1992). La dimensión individual también tiene una única característica dinámica, que la definimos como expectativas. Estas expectativas representan una esperanza con respecto a alguna cosa (p.e. aprobar un examen de cálculo porque se me da muy bien). La gente tiende a comportarse de una manera que su comportamiento encaje con sus expectativas, y por tanto, el conocimiento de las expectativas de un estudiante también es útil para adaptar el diseño de los programas de aprendizaje o de las metodologías (Sander et al. 2000). Por ejemplo, si los profesores conocen lo que esperan sus estudiantes, podrán ser capaces de adaptar su comportamiento a las expectativas de ellos, lo cuál debería tener un impacto positivo en los niveles de satisfacción de los mismos (Voss et al. 2007).

La dimensión del entorno define el conjunto de características que envuelven al contexto de aprendizaje, incluyendo una serie de características estáticas como la duración de la clase o los descansos entre asignaturas. Además, también establecemos un conjunto de características dinámicas que cambian más frecuentemente como la metodología que usa el profesor, el período lectivo en que se encuentra, los eventos planificados (exámenes, vacaciones, entregas, etc.), o las tareas que se realizan durante la clase (si son tareas realistas, estimulantes, adecuadas, etc.).

La dimensión social se caracteriza por un conjunto de características dinámicas que tienen que ver con la relación entre los individuos. El clima de la clase es uno de los factores que consideramos en el contexto social y se clasifican en: (a) consistentemente positivos y de apoyo, (b) consistentemente negativos y no apoyo, y (c) ambiguos. La relación entre alumno y profesor tiene un impacto en las emociones del alumno. Por ejemplo, los estudiantes y los profesores pueden crear un clima positivo que permita alinear sus objetivos (Schunk et al. 2002). La relación entre estudiante y grupo de compañeros es también importante e influye en los sentimientos de los estudiantes y en las emociones que emergen, tales como aquellas relacionadas con los logros y 


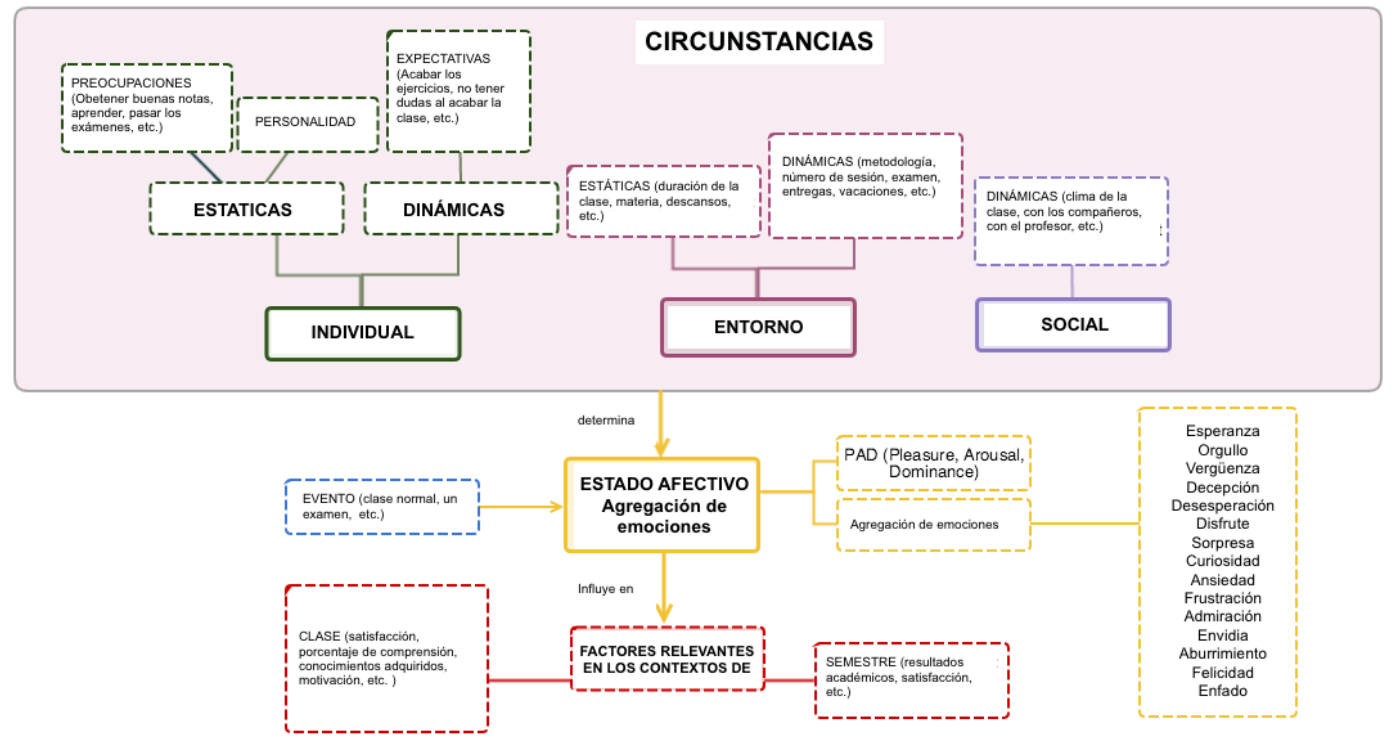

Fig. 1: Modelo que representa el estado anímico del estudiante.

fracasos, así como la aceptación y rechazo de otros (Graham 1991), o el aliniamiento con las aspiraciones académicas de los compañeros (Wang et al. 1997).

Estas tres dimensiones componen las distintas circunstancias que tienen un efecto directo con el estado anímico de un estudiante. La parte emocional de un estudiante tendrá un efecto directo en este estado anímico. Según (Meyer y Turner 2006), el estado anímico parece ser el mejor término para describir las emociones con el paso del tiempo. Nuestro modelo incluye dos maneras de establecer las emociones de los estudiantes: a través del modelo PAD (Placer, Excitación y Dominio), y como una agregación de emociones que relacionan estudiantes y entornos de aprendizaje. El estado anímico de un estudiante influye en los factores del contexto de aprendizaje. Estos factores se clasifican en el modelo como factores a corto plazo (satisfacción, porcentaje de comprensión, rapidez de adquisición de conocimientos y motivación) y factores a largo plazo (resultados académicos y satisfacción).

\section{Proceso}

A continuación describimos las partes del proceso que nos permite representar los distintos modelos que representan los estados anímicos de los estudiantes (Figura 2). Este proceso nos permite capturar los cambios de estados a través del tiempo y en función de las emociones que se provocan en las clases.

En la imagen podemos ver una serie de pasos requeridos para obtener información sobre los estudiantes. Esta información la recogemos en tiempo real durante las distintas clases. En un primer momento (identificado como Paso 1), los estudiantes rellenan una encuesta sobre las características estáticas que definen su dimensión individual (es decir, sobre sus intereses y su personalidad) y el profesor rellena la información 


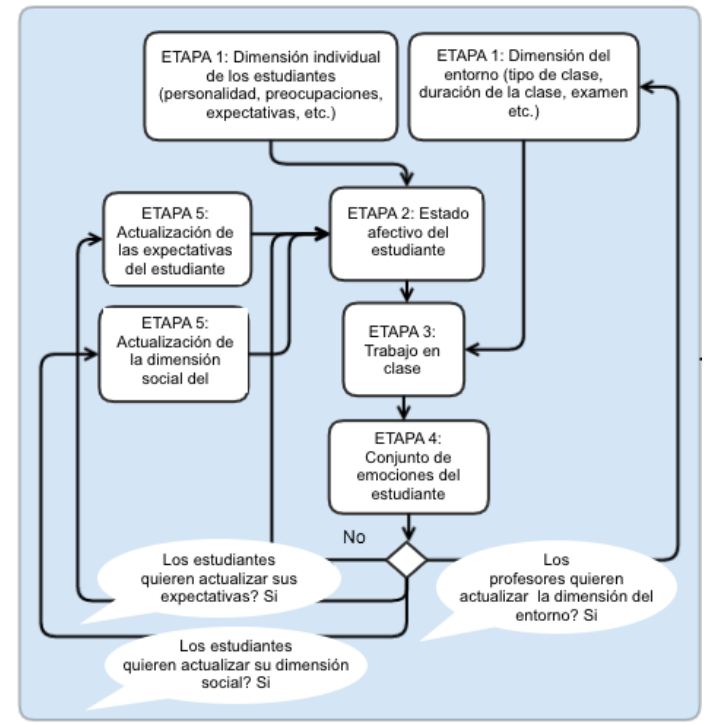

Fig. 2: Proceso para representar el estado anímico.

correspondiente a las características estáticas de la dimensión del entorno (es decir, la asignatura, duración de la clase, etc.). Como hemos comentado anteriormente, esta información permanece constante durante un período largo de tiempo y por tanto, se realiza una única vez al principio del proceso. Con respecto a las características dinámicas, bien al inicio de cada clase o bien al final de la misma, el profesor rellena (Paso 2) la información referente a las características dinámicas de la dimensión del entorno (metodología usada, fecha del calendario, proximidad a exámenes o períodos de vacaciones, etc.). Por su parte, los estudiantes rellenan al principio de cada clase (Paso 3) una encuesta respecto a las características dinámicas que definen su dimensión individual, es decir, sus expectativas para esa propia clase (terminar las tareas, no aburrirse, etc.) así como su estado afectivo mediante el modelo PAD. El Paso 3 está asociado al tiempo en el cuál se desarrolla la clase. Una vez la clase termina, en el Paso 4 los estudiantes rellenan otra encuesta con la información referente a su estado afectivo mediante un conjunto de emociones que sienten en ese momento. Después, antes de que tenga lugar la siguiente clase, los estudiantes pueden modificar la información que define su dimensión social (Paso 5). Finalmente, el profesor también puede modificar aspectos de la dimensión del entorno para observar cómo afectan los cambios del entorno en el estado afectivo de los estudiantes. La información recogida durante un período de tiempo, puede usarse para realizar simulaciones. 


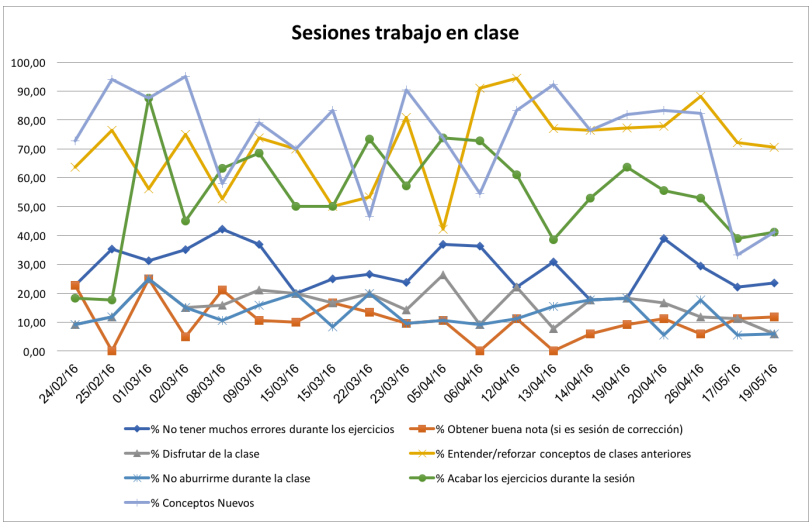

Fig. 3: Análisis temporal sobre las preocupaciones de los alumnos antes de empezar una clase de sesión de trabajo.

\section{Evaluación}

En esta sección presentamos la validación del modelo aplicado a tres asignaturas durante el curso 2015-2016. Los alumnos (20 alumnos de media en cada clase), durante un periodo aproximado de dos meses (65 clases) realizaron un test al inicio y al final de cada sesión. El test del inicio recogía información sobre sus expectativas y sobre sus preocupaciones antes de comenzar la clase. Hay que remarcar que las preocupaciones dependerán del estado en que esté el alumno en ese momento (p.e., si un alumno va mal en una asignatura, puede estar preocupado por reforzar los conceptos anteriores, mientras que si un alumno va bien, puede estar preocupado por disfrutar de la clase). El test del final recogía información sobre las emociones que tenían al acabar la clase.

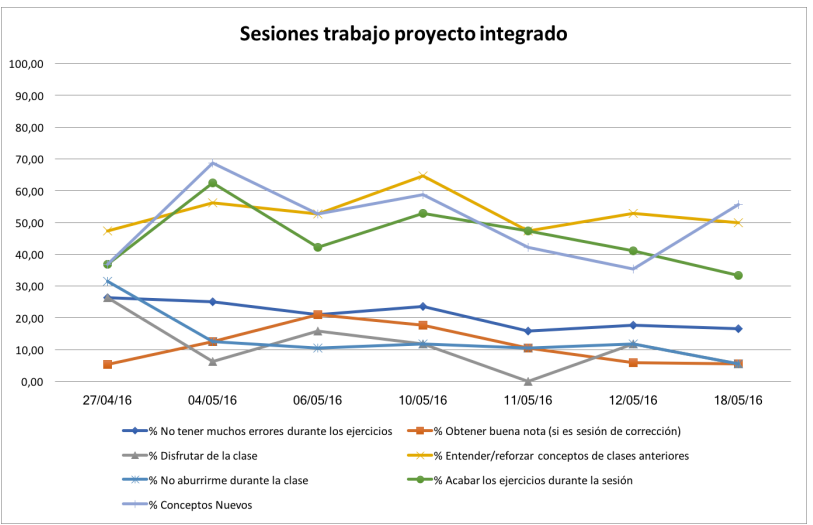

Fig. 4: Análisis temporal sobre las preocupaciones de los alumnos antes de empezar una clase de proyecto.

A partir de esta información, hemos analizado la evolución de las preocupaciones de los alumnos en distintos tipos de clase (e.g., trabajo en clase, trabajo por proyectos, repaso, y exámenes). También hemos analizado qué es lo que más les preocupaba a los alumnos a lo largo de los dos meses. Por otro lado, hemos analizado cómo han 


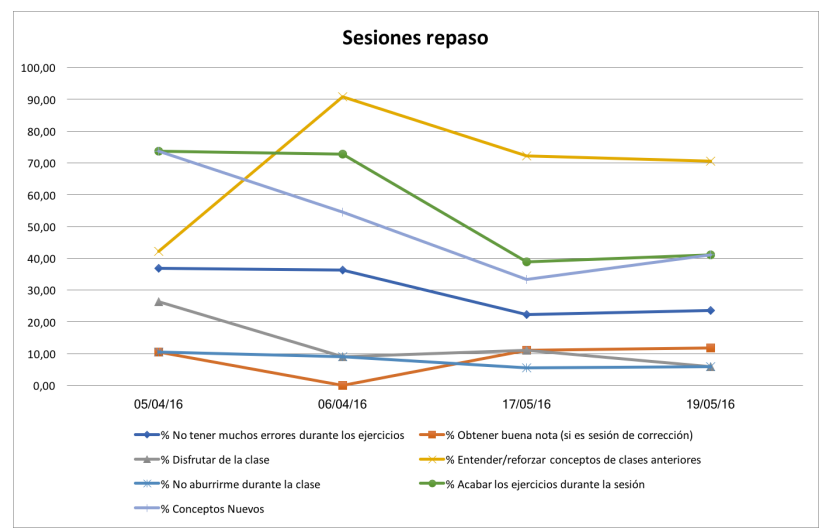

Fig. 5: Análisis temporal sobre las preocupaciones de los alumnos antes de empezar una clase de repaso.

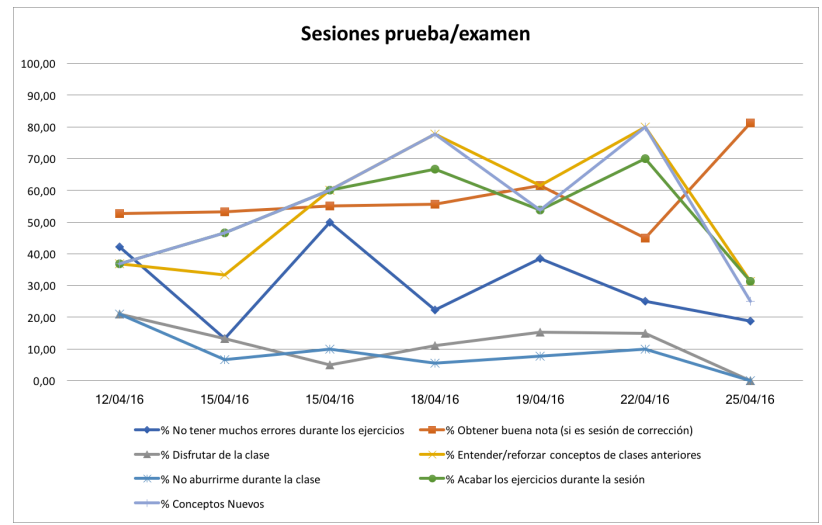

Fig. 6: Análisis temporal sobre las preocupaciones de los alumnos antes de empezar una clase de prueba/examen.

evolucionado las emociones positivas y negativas en los tipos de clase mencionados anteriormente y qué emociones, en promedio, han tenido los alumnos.

Las Figuras 3, 4, 5 y 6 muestran la evolución de las preocupaciones de los alumnos antes de empezar las clases. En las gráficas se puede observar que lo que más preocupaba a los alumnos en las sesiones de trabajo en clase de forma conjunta era entender y reforzar conceptos de clases anteriores, no aburrirse durante la clase y acabar los ejercicios. En sesiones de trabajo por proyectos las preocupaciones son similares a las clases donde se trabajaba de forma conjunta con el profesor pero el grado de preocupación es mucho menor. En el caso de sesiones de repaso se ve un claro cambio en las preocupaciones de los alumnos. En estas clases lo que más les preocupó a los alumnos era entender/reforzar los conceptos de las clases anteriores. Por último, en las clases donde hubo algún tipo de prueba o examen obtener buena nota era su principal preocupación junto con acabar los ejercicios. 


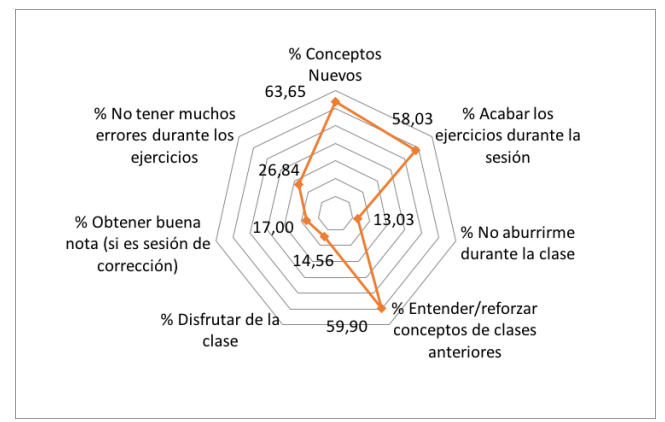

Fig. 7: Preocupación media de los alumnos antes de empezar la clase.

En general, las principales preocupaciones de los alumnos son aprender conceptos nuevos, entender y/o reforzar conceptos de clases anteriores, y acabar los ejercicios durante la sesión (ver Figura 7).

En las Figuras 8, 9, 10 y 11 se muestra la evolución de las emociones positivas y negativas en los distintos tipos de clase. En las clases de trabajo en grupo con el profesor se observa que en general las emociones son positivas, superiores al $50 \%$. Algo similar sucede en las sesiones de trabajo por proyectos donde las emociones positivas están alrededor del $70 \%$. En las sesiones donde hubo un examen o una prueba las emociones son en general positivas excepto en la última clase. Las clases de repaso son las que mejores emociones positivas generan estando muy cerca del $90 \%$.

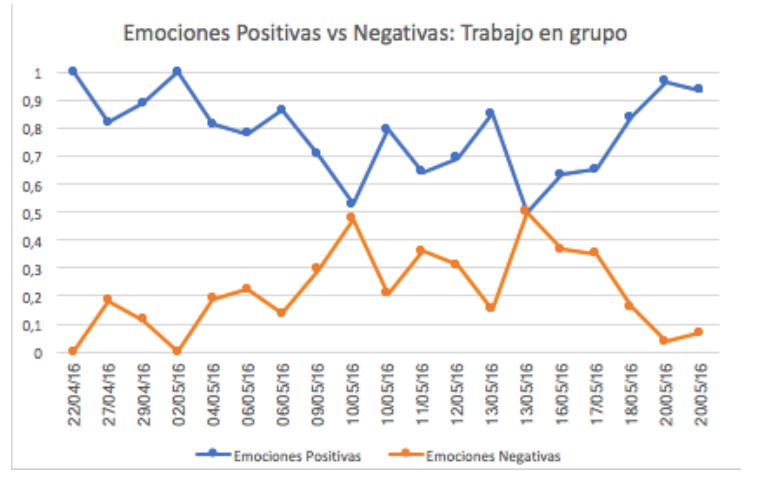

Fig. 8: Análisis temporal sobre las preocupaciones de los alumnos al acabar clases de trabajo.

Dentro de las emociones positivas y negativas, la Figura 12 muestra cómo se distribuyen en promedio en las emociones consideradas. En el caso de las emociones positivas, la emociones más predominantes son la esperanza (24\%) y la curiosidad $(26 \%)$. En el caso de las emociones negativas, la más predominante es la frustración (33\%). El aburrimiento es la siguiente emoción negativa más presente $(16 \%)$. 


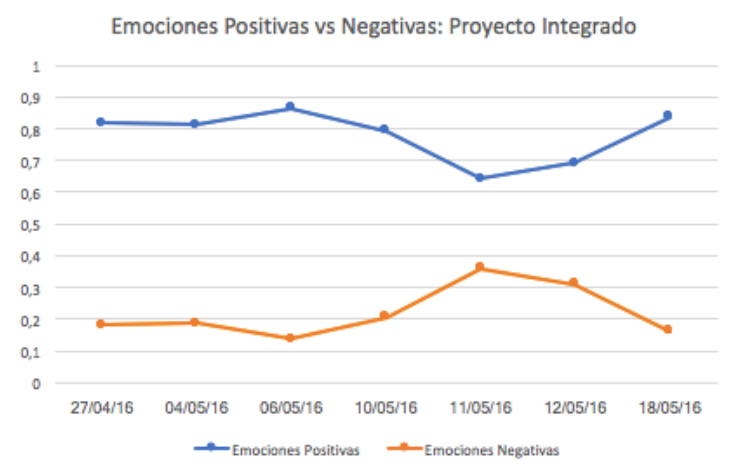

Fig. 9: Análisis temporal sobre las preocupaciones de los alumnos al acabar clases de proyectos.

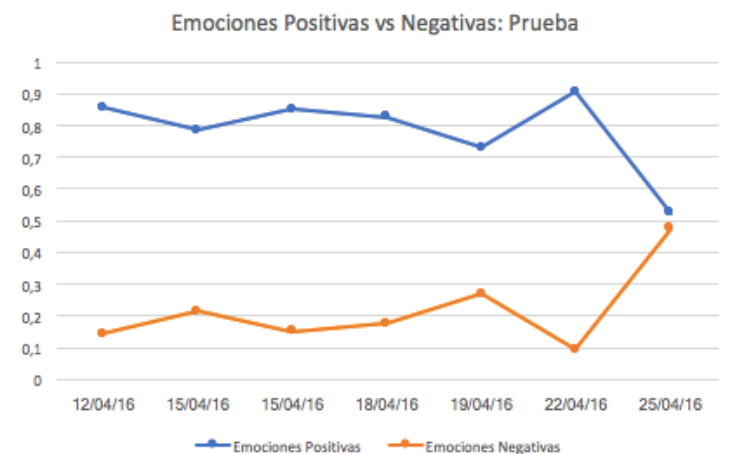

Fig. 10: Análisis temporal sobre las preocupaciones de los alumnos al acabar clases de prueba/examen.

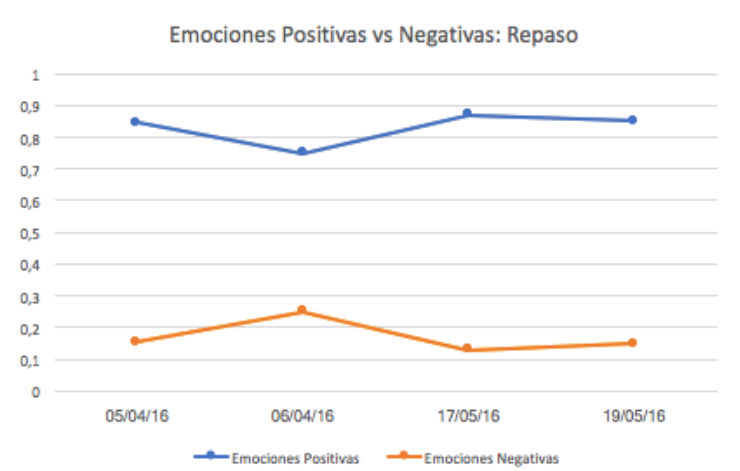

Fig. 11: Análisis temporal sobre las preocupaciones de los alumnos al acabar clases de repaso. 


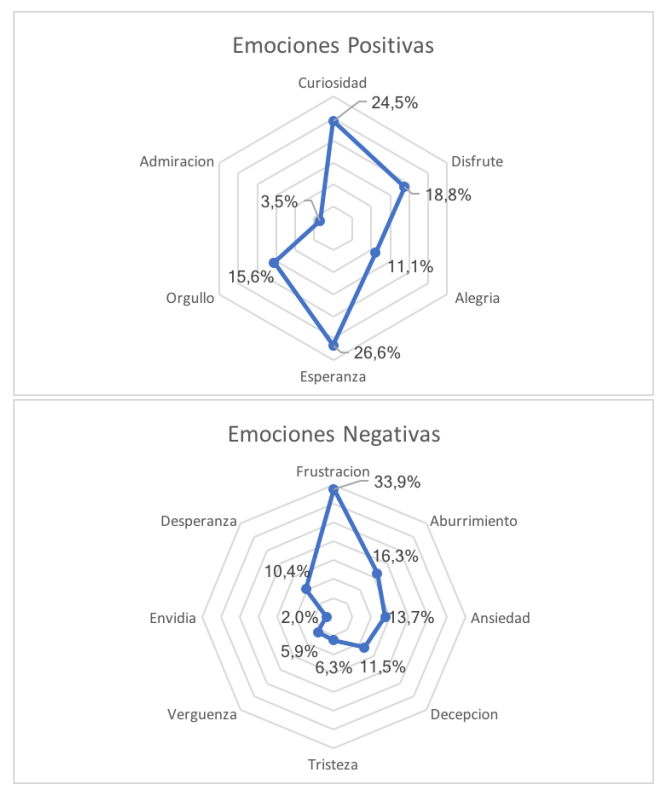

Fig. 12: Preocupación media de los alumnos al acabar la clase.

\section{Conclusiones}

Las emociones desempeñan un papel importante en los entornos de aprendizaje. En este artículo proponemos un modelo que considera un conjunto de factores y eventos desde una perspectiva temporal multi-nivel para anlizar su influencia en las emociones del estudiante. Esta perspectiva multi-nivel temporal considera tres dimensiones que incluyen características estáticas y dinámicas: individuales (preocupaciones, personalidad y expectativas), ambiental o del entorno (características relacionadas con la planificación y organización de la clase) y sociales (el clima de clase, la relación alumnos-compañeros, y la relación profesor-alumno). Este conjunto de características y los acontecimientos que pueden ocurrir durante una clase, influyen en las emociones del estudiante, y por lo tanto, en los factores de aprendizaje esperados tales como satisfacción, adquisición del conocimiento, motivación o comprensión. Basándonos en este modelo, hemos realizado un conjunto de encuestas para analizar la influencia de algunos de los aspectos considerados en el modelo. Los resultados nos muestran que la preocupación principal, independientemente del tipo de clase, es entender los conceptos explicados en clases anteriores y los nuevos y acabar los ejercicios.

La experiencia podría ser replicable a otros escenarios distintos (titulaciones, asignaturas, etc.) puesto que el proceso utilizado es independiente y el modelo permite capturar esta información específica. Por otro lado, el trabajo por proyecto y las clases de repaso son las clases que generan emociones más positivas. Como trabajo futuro, nos planteamos incluir más información del modelo para poder completar el análisis y en base a éste poder realizar simulaciones que nos ayuden a la toma de decisiones en el diseño de las clases. 


\section{Referencias bibliográficas}

P. Chalfoun, S. Chaffar, and C. Frasson, Predicting the emotional reaction of the learner with a machine learning technique,in ITS06, 2006.

P. T. Costa and R. R. McCrae, Four ways five factors are basic, Personality and individual differences, vol. 13, no. 6, pp. 653-665, 1992.

S. Graham, A review of attribution theory in achievement contexts, Educational Psychology Review, vol. 3, no. 1, pp. 5-39, 1991.

N. Ibrahimoglu, I. Unaldi, M. Samancioglu, and M. Baglibel, The relationship between personality traits and learning styles: A cluster analysis, Asian Journal of Management Sciences and Education, vol. 2, no. 3, 2013.

R. Lazarus, Emotion and Adaptation. Oxford University Press, 1994.

A. Mehrabian, Pleasure-arousal-dominance: A general framework for describ- ing and measuring individual differences in Temperament, Current Psychology, vol. 14, no. 4, pp. 261-292, 1996.

D. K. Meyer and J. C. Turner, Re-conceptualizing emotion and motivation to learn in classroom contexts, Educational Psychology Review, vol. 18, no. 4, pp. 377-390, 2006.

A. Ortony, G. L. Clore, and A. Collins, The Cognitive Structure of Emotions. Cambridge University Press, July 1988.

R. Pekrun, A. C. Frenzel, T. Gotz, and R. P. Perry, The Control-Value Theory of Achievement Emotions: an Integrative Approach to Emotions in Education, in Emotion in education, pp. 13-36, Academic Press, 2007.

R. M. Ryckman, Theories of Personality. PSY 235 Theories of Personality Series, Thomson/Wadsworth, 2007.

P. Sander, K. Stevenson, M. King, and D. Coates, University students expectations of teaching, Studies in Higher education, vol. 25, no. 3, pp. 309-323, 2000.

K.R.Scherer, Appraisal considered as aprocess of multilevel sequential checking, Appraisal processes in emotion: Theory, methods, research, vol. 92, p. 120, 2001.

D. Schunk, P. Pintrich, and J. Meece, Motivation in Education: Theory, Research, and Applications. Pearson/Merrill Prentice Hall, 2008.

R. Voss, T. Gruber, and I. Szmigin, Service quality in higher education: The role of student expectations, Journal of Business Research, vol. 60, no. 9, pp. 949-959, 2007.

M. C. Wang, G. D. Haertel, and H. J. Walberg, What Helps Students Learn? Spotlight on Student Success., 1997. 